\title{
Statistical Analysis of Harmony Search Algorithms in Tuning PID Controller
}

\author{
Salem Mohammed $^{1 *}$, Khelfi Mohamed Fayçel ${ }^{2}$, Bachir Bouiajra Rochdi ${ }^{3}$ \\ ${ }^{1,3}$ Computer Science Departments, University of Mustapha Stambouli, Mascara, Algeria \\ ${ }^{2}$ Computer Science Department, University of Ahmed Ben Bella 1, Oran, Algeria \\ * Corresponding author's Email: salemohammed@gmail.com
}

\begin{abstract}
Harmony search (HS) is a new meta-heuristic optimization method inspired from improvisation process in jazz music searching for a perfect state of harmony. HS has been improved based on parameters settings updating and hybridization. In this paper, HS and four of its variants are compared via Wilcoxon and Friedman nonparametric tests using benchmark functions. Statistical analysis highlights the Global best harmony search as the best variant. Harmony Search algorithm and its variants are also applied to adjust the gains of PID controller of an inverted pendulum nonlinear system giving remarkable results when compared to genetic algorithm.

Keywords: Harmony search; Heuristic optimization; harmony search variants; Wilcoxon test; Friedman test; Statistical Analysis; Musical Improvisation; PID control; Inverted pendulum.
\end{abstract}

\section{Introduction}

Analytical methods are widely used to solve optimization problems giving an exact solution to minimize or maximize a given function by verifying a set of constraints.

In some cases, the problem sizes may hinder the calculation efficiency and/or the solution is obtained in very large time. These drawbacks could be figured out by using meta-heuristics.

Meta-heuristics are nature inspired optimization methods, easy to implement to different problems and although aiming to find a high quality solution, they cannot pretend to produce the exact solution in every case with certainty. Nevertheless, a high quality approximation of a global optimum is probably more valuable than a deterministic poor quality local minimum provided by an exact method or no solution at all. [1].

As free lunch theorem suggests [14], there is no best algorithm for all problems but the variety of these algorithms could lead ones to decide for the best one in optimization of problems with partial knowledge, which is not a trivial task. This decision could be taken using experimental analysis and graphical comparison but the result is not reliable and could not be generalized [5, 12].

Statistical inference could be used in the analysis of empirical results obtained by the algorithms. It shows how well a sample of results satisfy a certain hypothesis and if the obtained conclusions can be generalized beyond what was tested [18].

The Harmony Search (HS) algorithm is an emerging optimization meta-heuristic inspired from the underlying musicians harmony improvisation process [3, 4]. When musicians want to compose a harmony, they usually try various possible combinations of musical pitches stored in their memories. Harmony Search algorithm had been applied very successful in a variety of optimization problems ([5],[7],[6], [9]).

In HS algorithm, diversification is achieved by generating a new solution after considering all of the existing vectors and both pitch adjustment and random consideration. These features increase the flexibility of HS algorithm and produce better solutions.

Recently, the interest in harmonic search algorithm has led the researchers to enhance and develop new variants exploring two aspects: improvement of parameters settings, and hybridizing 
HS with other meta-heuristics. Among these interesting variants, we will focus over the Improved harmony search algorithm (IHS) [7], Global-best harmony search (GHS) [8], Self-adaptive Harmony Search (SAHS) [9] and Adaptive Harmony Search (AHS) [10].

In this paper, statistical analysis is achieved over harmony search and its variants to extract reliable and generalized conclusion on the best harmony search variant in function optimization in order to find the optimal gains of a PID controller.

The conditions of safe use of parametric tests have not been satisfied so nonparametric are carried out in single and multiple problem analysis with Wilcoxon and Friedman tests. This analysis will be conducted in two steps:

First, the original HS algorithm and the four variants will be used to minimize known benchmark functions and compared statistically using the Wilcoxon and Friedman nonparametric tests.

Second, real world application is also presented where the harmonic search optimization algorithms are applied in an optimization via simulation process to determine an optimum structure of PID controller in order to have the best performance of an inverted pendulum.

The remainder of this paper is organized as follows: In section II, harmony search algorithm is detailed and four of its variants are presented in section III. Results of statistical analysis using benchmark functions and nonparametric tests are highlighted in section IV. Section V is dedicated to design a tuning procedure to adjust the PID controller gains. Simulations results are shown in section VI where the harmony search algorithm and its variants are compared to genetic algorithm to adjust the PID controller for an inverted pendulum.

\section{Harmony search optimization}

The Harmonic search algorithm is a new metaheuristic based on typical music performance processes that occur when musicians are seeking for better state of harmony, as for improvisation in jazz. It was developed by [4] who conceptualized the use of musical process for a perfect state of harmony.

The optimization process can be compared to a process of improvisation of jazz musician [3]. The details of the analogy between musical improvisation and optimization are shown in Figure. 1.

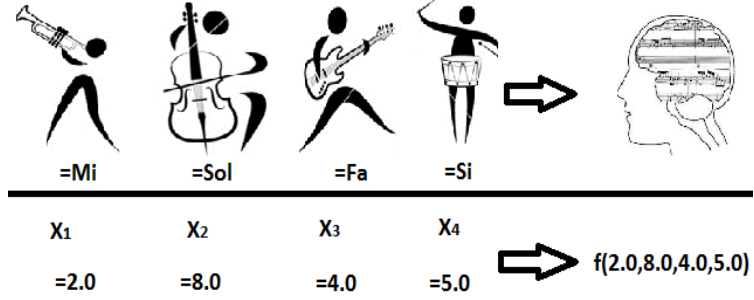

Figure.1 Analogy between musical improvisation and optimization

\subsection{Harmony search parameters settings}

An optimization problem could be written as follows:

$$
\begin{gathered}
\operatorname{Min} f\left(x_{i}\right) \\
x_{i} \in S_{i}, i=1,2, \ldots N
\end{gathered}
$$

where $f\left(x_{i}\right)$ is the function to be optimized, $x_{i}$ are the $\mathrm{N}$ decision variables.

The decision variables and their corresponding objective function values are stored in a matrix called Harmony memory. The HMCR and PAR parameters are used to improve the solution and ensure intensification and diversification processes respectively.

The computational procedure of HS is described in Figure 2 [4] and the parameters of the algorithm are presented in Table I.

\subsection{Initializing the harmony memory (HM)}

After setting the Harmony search parameters, the harmony memory matrix, shown in (2), is initialized by random vector solutions and evaluated using the objective function.

Table 1. Harmony search parameters

\begin{tabular}{|c|l|}
\hline Parameter & \multicolumn{1}{|c|}{ Description } \\
\hline $\mathrm{NI}_{\max }$ & Improvisations Max number. \\
$\mathrm{HMS}$ & Harmony Memory Size \\
$\mathrm{HMCR}$ & Harmony Memory considering Rate. \\
$\mathrm{PAR}$ & Pitch Adjusting Rate \\
$\mathrm{bw}$ & Distance bandwidth \\
$\mathrm{x}^{\mathrm{I}}, \mathrm{x}^{\mathrm{S}}$ & Lower and higher bounds of decision \\
& variables \\
\hline
\end{tabular}




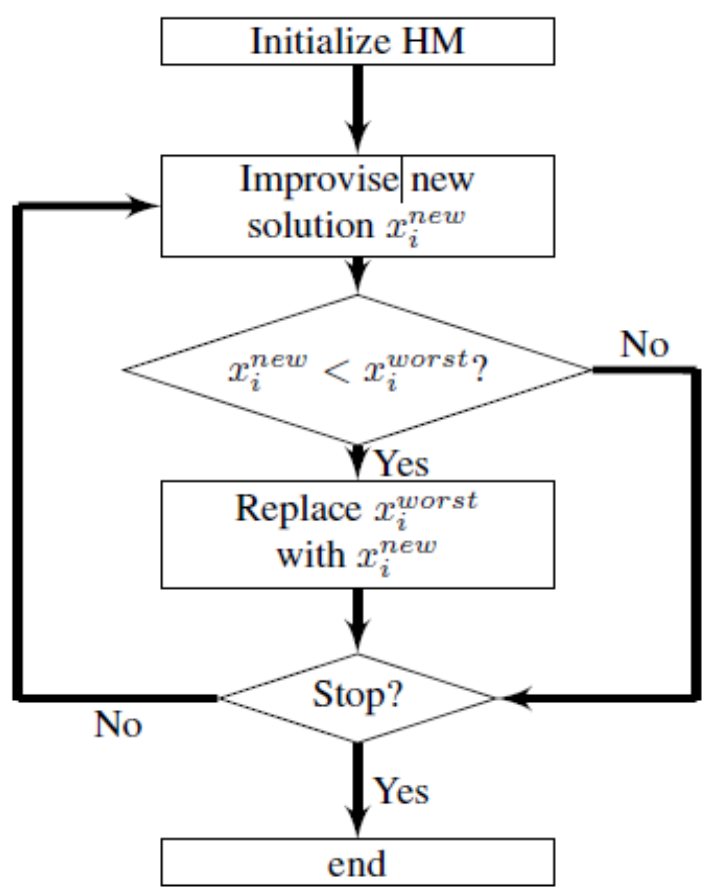

Figure.2 Harmony search flowchart

$$
H M=\left[\begin{array}{c}
x^{1} \\
x^{2} \\
\cdot \\
\cdot \\
\cdot \\
x^{H M S}
\end{array}\right]=\left[\begin{array}{ccccc}
x_{1}^{1} & x_{2}^{1} & \ldots . x_{N}^{1} & f\left(x^{1}\right) \\
x_{1}^{2} & x_{2}^{2} \ldots & x_{N}^{2} & f\left(x^{2}\right) \\
& \cdot & \cdot & & \cdot \\
& & & \\
x_{1}^{H M S} & x_{2}^{H M S} & \ldots & x_{N}^{H M S} & f\left(x^{H M S}\right)
\end{array}\right]
$$

where $x^{j}$ is the $j^{\text {th }}$ solution vector in the harmony memory and $\mathrm{x}_{\mathrm{i}}^{\mathrm{j}}$ is the $\mathrm{i}^{\text {th }}$ variable in $\mathrm{x}^{\mathrm{j}}$ [4].

\subsection{Improvisation of a new harmony}

In Jazz improvisation, a musician plays a note, he selects it randomly from the total playable range (see Figure.3), memory of the musician (see Figure. 4) or by tweaking the note obtained from its memory (see Figure.5). In the same way, the HS algorithm improvises a value by choosing it from the total values range, from HM or by or tweaking the value which was originally chosen from HM.

\subsubsection{Random selection}

The $\mathrm{i}^{\text {th }}$ decision variable value of the new solution $\mathrm{x}^{\text {new }}$ could be chosen randomly where $\mathrm{x}_{\mathrm{i}}^{\mathrm{I}}$ $\leq \mathrm{x}_{\mathrm{i}}^{\text {new }} \leq \mathrm{x}_{\mathrm{i}}^{\mathrm{S}}$ with probability $1-H M C R$ like in (3). Random selection is also used for start harmony memory initialization. if $($ rand $\in(0,1) \geq H M C R)$ then

$$
x_{i}^{\text {new }}=\operatorname{rand}\left(x_{i}^{I}, x_{i}^{S}\right)
$$

$x_{i}^{I}$ and $x_{i}^{S}$ are the lower and higher boundary values of decision variables.

\subsubsection{Memory consideration}

Harmony search algorithm could choose the new solution $\mathrm{x}^{\text {new }}$ by randomly selecting it from the harmony memory with probability (HMCR) using (4).

$$
\left\{\begin{array}{c}
\text { if }(\text { rand } \in(0,1) \leq H M C R) \text { then } \\
\mathrm{x}^{\text {new }} \in H M
\end{array}\right.
$$

\subsubsection{Pitch adjustment}

After selecting the new solution $\mathrm{x}^{\text {new }}$ from HM by Memory Consideration, it could be adjusted or not according to its neighbouring values with probability (PAR) by adding a random value using (5) [3].

$$
\begin{gathered}
\text { if }(\text { rand } \in(0,1) \leq P A R) \text { then } \\
x_{i}^{\text {new }}=x_{i}^{\text {new }}+b w * \operatorname{rand}(-1,1)
\end{gathered}
$$

where bw is the distance bandwidth.

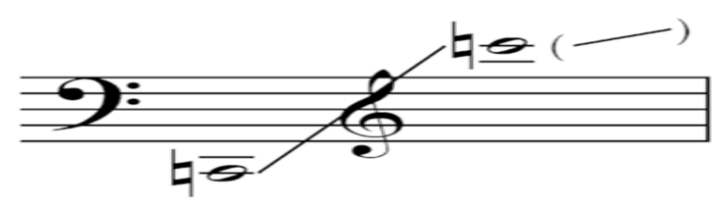

Figure 3. Playable ranges of a musical instrument

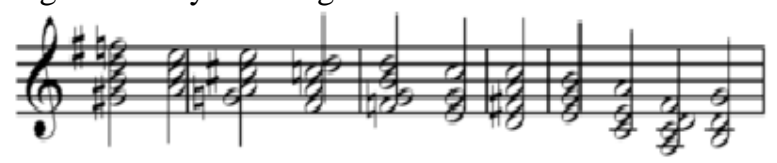

Figure 4. Good notes in musician's memory

OLD:

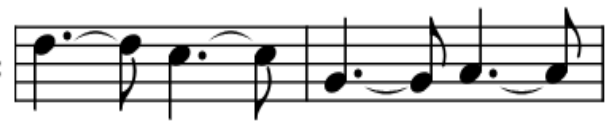

NEW:

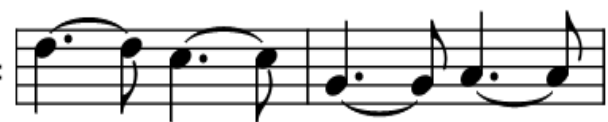

Figure 5. Tweaking the note from musician's memory 


\subsubsection{Updating Harmony Memory}

If the new harmony $\mathrm{x}^{\text {new }}$ is better than the worst vector in HM, the new harmony replaces the worst one in the HM [3].

$$
\begin{gathered}
\text { if } f\left(x^{\text {new }}\right)<\mathrm{f}\left(x^{\text {worst }}\right) \text { then } \\
H M(\text { worst },:)=x^{\text {new }}
\end{gathered}
$$

\section{Variants of harmonic search algorithm}

Good parameter values selection is considered one of the challenges, not only for the HS algorithm, but also for other meta-heuristics. This challenge has led researchers to develop some interesting variants for the harmony search by improving the parameters settings or by hybridization with other metaheuristics. In the next section, four of these variants are presented.

\subsection{Improved harmony search algorithm (IHS)}

A very well-known harmony search improvement is given by Mahdavi and known as Improved Harmonic Search [7]. They proposed to improve the original algorithm by a dynamic adaptation of the adjustment rate values (PAR) and bandwidth (bw). PAR value is increased with each improvisation $t$ in IHS algorithm using (7):

$$
P A R_{t}=P A R_{\min }+t \frac{\left(P A R_{\max }+P A R_{\min }\right)}{N I}
$$

where $P A R_{t}$ is the value of PAR in the $t^{t h}$ improvisation, and $P A R_{\min }$ and $P A R_{\max }$ are the minimum and the maximum pitch adjusting rate (PAR).

The pitch value bw is dynamically updated for each improvisation as follows:

$$
b w_{t}=t b w_{\text {max }} e^{\left(\frac{\ln \left(\frac{b w_{\min }}{b w_{\max }}\right)}{N I}\right)}
$$

$b w_{\min }$ and $b_{\max }$ are the minimum and the maximum pitch values.

\subsection{Global-best harmony search (GHS)}

Another major improvement made by Omran and Mahdavi [8] called Global best harmony search (GHS). It is inspired from the PSO optimization; it modifies the pitch-adjustment step of the HS where the new Harmony is chosen as the best harmony in the HM, so the bw parameter is eliminated, as illustrated in (9).

$$
\begin{aligned}
& \text { if }(\text { rand } \in(0,1) \leq P A R) \text { then } \\
& x_{k}^{\text {new }}=x_{k}^{\text {best }} / k \in \operatorname{rand}(1, N)
\end{aligned}
$$

\subsection{Self-adaptive harmony search (SAHS)}

Wang and Huang [9] proposed the Self-adaptive Harmony Search, (SAHS), with automatically adjusted parameters. Indeed, the pitch-adjustment step is modified so the new harmony is updated with either (10) or (11) with equal probability:

$$
\begin{gathered}
x_{i}^{\text {new }}=x_{i}^{\text {new }}+\left[\max \left(H M_{i}\right)-x_{i}^{\text {new }}\right] \operatorname{rand}(0,1) \\
x_{i}^{\text {new }}=x_{i}^{\text {new }}+\left[x^{i}-\min \left(H M_{t}\right)\right] \operatorname{rand}[0,1]
\end{gathered}
$$

where $\operatorname{rand}(0,1)$ is a randomly generated value between 0 and $1, \mathrm{x}_{\mathrm{i}}^{\text {new }}$ is the $i^{\text {th }}$ variable of the new harmony, $\min \left(\mathrm{HM}_{\mathrm{t}}\right)$ and $\max \left(\mathrm{HM}_{\mathrm{t}}\right)$ are the minimum and the maximum values of the HM in the $t^{\text {th }}$ improvisation.

\subsection{Adaptive harmony search}

Hasancebi [10] proposed a new adaptive algorithm where HMCR and PAR parameters are assigned dynamically during improvisation process by probabilistically selecting them around average values of these parameters observed within the current harmony memory matrix using (12) and (13).

$$
\begin{gathered}
H_{M C R_{t}}=\left(1+\frac{1-H M C R_{0}}{H M C R_{0}} \cdot e^{-\gamma \cdot N(0,1)}\right)^{-1} \\
P A R_{t}=\left(1+\frac{1-P A R_{0}}{P A R_{0}} \cdot e^{-\gamma \cdot N(0,1)}\right)^{-1}
\end{gathered}
$$

$H M C R_{t}$ and $P A R_{t}$ represent the sampled values of the control parameters for the new harmony vector. $\mathrm{N}(0,1)$ is the normally distributed random number having expectation 0 and standard deviation 1 . The symbols $H M C R_{0}$ and $P A R_{0}$ denote the average values of control parameters within the harmony memory matrix, obtained by averaging the corresponding values of all the solution vectors within the HM matrix, that is:

$$
\left\{\begin{aligned}
H M C R_{0} & =\frac{\sum_{i=1}^{H M S} H M C R_{i}}{H M S} \\
P A R_{0} & =\frac{\sum_{i=1}^{H M S} P A R_{i}}{H M S}
\end{aligned}\right.
$$


The factor $\gamma$ refers to the learning rate of control parameters, which is recommended to be selected within a range of [0.25 0.50].

\section{Statistical analysis of the harmony search algorithm and its variants}

\subsection{Experimental settings}

To evaluate the performances of Harmony search algorithm and its variants, statistical tests will be achieved in order to compare them. Indeed, Harmony search (HS), improved harmony search algorithm (IHS), Global-best harmony search (GHS), Adaptive Harmony Search (AHS) and Self-adaptive Harmony Search (SAHS) are used to find the minimum of 10 benchmark functions listed as follows [11]:

- F1: Sphere function.

$$
f\left(x_{1} . . x_{D)}=\frac{1}{D} \sum_{i=1}^{D} x_{i}^{2}\right.
$$

- F2: Ackley function

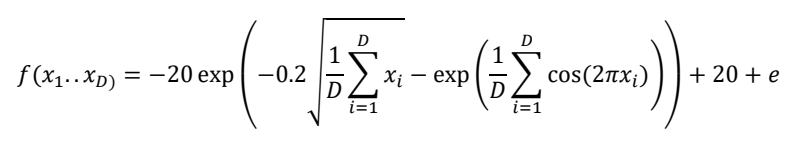

- F3: Ridge function

$$
f\left(x_{1} . . x_{D}\right)=\sum_{i=1}^{D}\left(\sum_{j-1}^{i} x_{j}\right)^{2}
$$

\section{- F4: Rosenbrock function}

$$
f\left(x_{1} . . x_{D}\right)=\frac{1}{D} \sum_{i=1}^{D-1}\left(100\left(x_{i}^{2}-x_{i-1}\right)^{2}-\left(1-x_{i}\right)^{2}\right)
$$

- F5: Schwefel function

$$
f\left(x_{1} . . x_{D}\right)=\sum_{i=1}^{D}\left(-x_{i}-\sin \left(\sqrt{\left|x_{i}\right|}\right)\right)+\alpha . D
$$

- F6: Rastrigin function

$$
f\left(x_{1} . . x_{D}\right)=10 D+\sum_{i=1}^{D}\left(x_{i}^{2}-10 \cos \left(2 \pi x_{i}\right)\right)
$$

\section{- F7: Trid functions}

$$
f\left(x_{1} . . x_{D)}=\sum_{i=1}^{D}\left(x_{i}-1\right)^{2}-\sum_{i=1}^{D} x_{i} x_{i-1}\right.
$$

- F8: Sum squared function

$$
f\left(x_{1} . . x_{D)}=\sum_{i=1}^{D} i x_{i}^{2}\right.
$$

- F9: Power Sum function

$$
f\left(x_{1} . x_{D}\right)=\sum_{i=1}^{D}\left[\left(\sum_{j=1}^{D} x_{j}^{i}\right)-b_{i}\right]^{2}
$$

\section{- F10: Griewank function}

$$
f\left(x_{1} . . x_{D}\right)=1+\frac{1}{4000} \sum_{i=1}^{D} x_{i}^{2}-\prod_{i-1}^{D} \cos \left(\frac{x_{i}}{\sqrt{i}}\right)
$$

To carry out the comparison, each algorithm is run 30 independent runs to minimize each problem with dimension $\mathrm{D}=10$. The termination criterion is either when the maximum evaluations number: $100^{\mathrm{D}}$ is reached or the error value is smaller than $10^{-4}$.

The parameters of the compared algorithms are in Table. 2 and average errors (for the minimum values obtained from the 30 runs) are in Table.3.

Statistical tests are usually used to draw inferences about one or more populations from given samples. Before carrying out a test, two hypotheses are defined, the null hypothesis which states the no difference between the compared populations whereas the alternative hypothesis represents the presence of difference [12]. When applied, a hypothesis testing requires a level significance $\alpha$ to determine at which level the hypothesis may be rejected. In our case we carried out two nonparametric tests due to the violation of data normality pre-condition in Table.3 [12].

Table 2. HS variants' parameters

\begin{tabular}{|l|c|c|c|c|c|}
\hline \multicolumn{1}{|c|}{ Parameter } & HS & GHS & IHS & AHS & SAHS \\
\hline $\mathrm{NI}_{\max }$ & 20 & 20 & 20 & 20 & 20 \\
$\mathrm{HMS}$ & 3 & 3 & 3 & 3 & 3 \\
$\mathrm{HMCR}$ & 0.995 & 0.995 & 0.995 & $/$ & 0.995 \\
$\mathrm{PAR}$ & 0.01 & 0.01 & $/$ & $/$ & 0.01 \\
$\mathrm{bw}$ & 0.01 & $/$ & $/$ & $/$ & $/$ \\
$\gamma$ & $/$ & $/$ & $/$ & 0.3 & $/$ \\
PAR $_{\max }$ & $/$ & $/$ & 0.1 & $/$ & $/$ \\
PAR $_{\min }$ & $/$ & $/$ & 0.001 & $/$ & $/$ \\
bw $_{\max }$ & $/$ & $/$ & 0.005 & $/$ & $/$ \\
bw $_{\min }$ & $/$ & $/$ & 0.001 & $/$ & $/$ \\
\hline
\end{tabular}

Table 3. Average errors obtained for the 10 benchmark functions.

\begin{tabular}{|c|c|c|c|c|c|}
\hline Num & \multirow{2}{*}{$\boldsymbol{H} \boldsymbol{S}$} & $\boldsymbol{G H S}$ & $\boldsymbol{H} \boldsymbol{S}$ & $\boldsymbol{S} \boldsymbol{H} \boldsymbol{S}$ & $\boldsymbol{I} \boldsymbol{H} \boldsymbol{S}$ \\
\hline F1 & 0.0000 & 0.000 & 0.000 & 0.000 & 0.000 \\
F2 & 0.0001 & 0.000 & 410.00 & 0.000 & 211.000 \\
F3 & 0.0201 & 0.066 & 1400.00 & 3100.00 & 3620.00 \\
F4 & 0.020 & 0.000 & 3.3297 & 0.000 & 1.690 \\
F5 & 0.000 & 0.000 & 47716.00 & 0.000 & 0.002 \\
F6 & 41.900 & 0.000 & 47.228 & 43.000 & 37.000 \\
F7 & 5.544 & 4.971 & 41.650 & 43.200 & 23.000 \\
F8 & 21.100 & 15.119 & 21.190 & 21.100 & 21.100 \\
F9 & 8.180 & 7.220 & 74.306 & 43.600 & 45.200 \\
F10 & 0.000 & 0.000 & 0.1047 & 0.024 & 0.850 \\
\hline
\end{tabular}




\subsection{Wilcoxon signed ranks tests}

The Wilcoxon signed ranks test is a nonparametric test that compares two paired groups (algorithms) in aim to detect significant differences between their behaviors.

It is considered as the nonparametric equivalent of the parametric t-test. The test essentially computes the difference between each set of pairs and analyses these differences. The Wilcoxon Rank Sum test can be used to accept (or reject) the null hypothesis that two samples represent two different populations [12].

The Wilcoxon test is carried out in pairwise comparison of the HS variants considered in the analysis. Table. 4 shows the $\mathrm{R}+, \mathrm{R}$ - and p-values computed for all pairs (the p-values have been obtained by using SPSS)[12]. As the table states:

- GHS shows a significant improvement over all the others algorithms with a level of significance $\alpha=0: 05$.

- AHS is clearly better than HS with $\alpha=$ 0.1 and from HIS with $\alpha=0: 05$.

- We can remark also that the hypothesis IHS is better than HS and SAHS are strongly rejected with level 0.139 and 0.0327 respectively.

\subsection{Friedman test}

The Friedman Test [16] is the non-parametric alternative to the one-way ANOVA with repeated measures. It is used to test for differences between groups in multiple comparisons with a control method. The null hypothesis for Friedman's test states the equality of medians between the populations.

We applied the Friedman test to compare GHS (control method) with the other four algorithms (HS, IHS, SAHS, AHS) with $\alpha=0: 05$. The results of the test are represented in Table 5. Friedman statistic (distributed according to chi-square with 4 degrees of freedom is 79.64795. while the p-value computed by Friedman Test is 17E-5. The null hypothesis (Equality of medians) is rejected by the Friedman test (p-value $<\alpha$ ), so we could conclude that there is a difference between the compared algorithms. As the null hypothesis is rejected, we can proceed with post-hoc tests to control the family-wise error in multiple hypotheses testing, such as Hommel, Li and Hochberg post-hoc procedure. The p-values of these three post-hoc procedures are shown in Table.6 [12].
Table 4. Wilcoxon signed ranks test results.

\begin{tabular}{|c|c|c|c|}
\hline Pair & R- & R+ & p-value \\
\hline GHS - AHS & 28 & 0 & 0.018 \\
GHS - HS & 45 & 0 & 0.008 \\
GHS - SAHS & 21 & 0 & 0.028 \\
GHS - IHS & 45 & 0 & 0.008 \\
AHS - SAHS & 25 & 3 & 0.063 \\
AHS - HS & 45 & 0 & 0.008 \\
AHS - IHS & 32 & 4 & 0.050 \\
SAHS - HS & 34 & 11 & 0.173 \\
IHS - SAHS & 11 & 25 & 0.327 \\
IHS -HS & 35 & 10 & 0.139 \\
\hline
\end{tabular}

Table 5. Average rankings by Friedman test

\begin{tabular}{|c|c|}
\hline Algorithm & Ranking \\
\hline HS & 1.6 \\
\hline SAHS & 3.099 \\
\hline IHS & 2.400 \\
\hline AHS & 3.75 \\
\hline GHS & 4.15 \\
\hline Statistic & 79.64795 \\
\hline pvalue & $17 \mathrm{E}-5$ \\
\hline
\end{tabular}

Table 6. Post-hoc procedures results, Friedman test

\begin{tabular}{|c|c|c|c|c|}
\hline Algorithm & AHS & SAHS & IHS & HS \\
\hline unadjusted p & $3.106 \mathrm{E}-4$ & 0.0023 & 0.0338 & 0.2578 \\
\hline Holm & 0.0012 & 0.0070 & 0.0677 & 0.2578 \\
\hline Li & $4.184 \mathrm{E}-4$ & 0.0031 & 0.0436 & 0.2578 \\
\hline Hochberg & 0.0012 & 0.0070 & 0.0677 & 0.2578 \\
\hline
\end{tabular}

\subsection{Discussion}

The statistical analysis has highlighted the GHS algorithm as the best one [16]. Indeed, Wilcoxon test has accepted all null hypotheses where GHS is better than each of the four compared algorithms.

Friedman test has confirmed these results by ranking GHS as the first one and when used as control method in the post-hoc procedures (Hommel, $\mathrm{Li}$ and Hockberg), all the null hypothesis were accepted. These performances are due to the hybridization with PSO which has led to improve the diversification of the algorithm.

The major drawback of the IHS is that the user needs to specify the minimum and maximum values for bw and PAR which are difficult to guess and problem dependent and it surely affects the performances of IHS in our case.

\section{PID controller tuning using harmony search algorithms}

PID control is a sum of three components, Proportional, Integral and Derivative part. It is used to cancel the error in (15) between the plant output $\mathrm{y}(\mathrm{t})$ and the desired output $\mathrm{y}_{\mathrm{d}}(\mathrm{t})$ [17]: 


$$
e(t)=y(t)-y_{d}(t)
$$

The control signal of the PID controller is given by (15):

$$
u(t)=K_{p} e(t)+K_{d} \dot{e}(t)+K_{i} \int e(t) d t
$$

where $K_{p}, K_{d}, K_{i}$ are constant positive gains to be determined.

Before using a PID controller we have to adjust its parameters. In case of nonlinear systems, tuning the gains is done usually by trial/error [15]. Adjusting the PID parameters could be considered as an optimization problem where ones try to find the optimal solution inside a predefined search space to fulfill a desired output of the nonlinear system [13].

The harmony search algorithm and its variants are used find the optimal combination of the PID controller parameters where each harmony is composed of the three gains (Figure 6).

Objective function in (17) is used to evaluate the harmonies of harmony search and its variants:

$$
f\left(K_{p}, K_{i}, K_{d}\right)=\left(1-e^{-\beta}\right)\left(O_{\max }+e_{s}\right)+e^{-\beta}\left(t_{r}-t_{m}\right)
$$

Where $t_{m}$ and $t_{r}$ are the settling time and rising time respectively. $\mathrm{O}_{\max }$ is the maximum authorized overshoot and $e_{s}$ is the steady state error while $\beta$ is a weighting factor to promote either the times $\left(t_{m}\right.$ and $\left.t_{r}\right)$ or errors $\left(e_{s}\right.$ and $\left.\mathrm{O}_{\max }\right)$,

\section{Simulation results}

Harmony search algorithm and its variants GHS (the best one from the statistical analysis) and IHS are compared to genetic algorithm (GA) and used in a real world application to find the optimal values of PID controller gains to control an inverted pendulum (See Figure.7).

The aim is to stabilize the pole angle $\theta\left(\theta_{d}=0\right)$. The initial parameters for the HS variants are in Table. 7 and those of GA are in Table. 8.

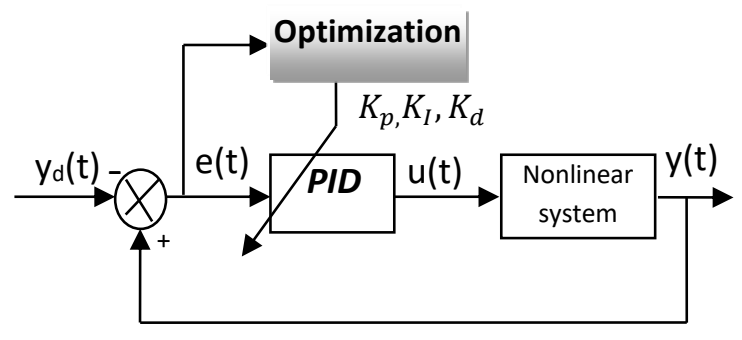

Figure 6. Tuning of PID controller using HS algorithms

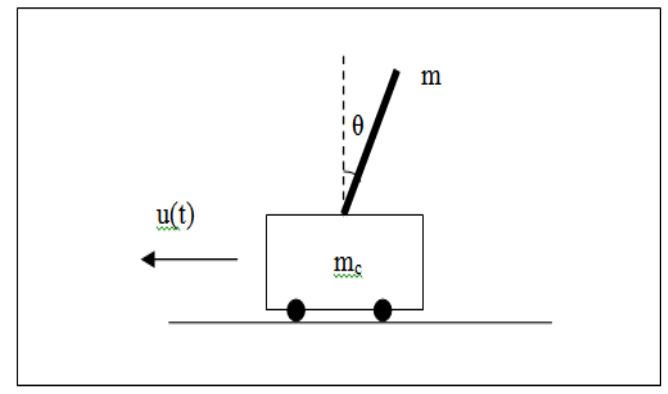

Figure 7. Inverted pendulum system

Table 7. Parameters settings for HS, GHS and IHS

\begin{tabular}{|c|c|c|c|}
\hline Parameter & HS & GHS & IHS \\
\hline $\mathrm{NI}_{\max }$ & 20 & 20 & 20 \\
$\mathrm{HMS}$ & 3 & 3 & $/$ \\
$\mathrm{HMCR}$ & 0.995 & $/$ & $/$ \\
$\mathrm{PAR}$ & 0.01 & $/$ & $/$ \\
$\mathrm{bw}$ & 0.01 & $/$ & $/$ \\
$\mathrm{x}^{\mathrm{I}}, \mathrm{x}^{\mathrm{S}}$ & {$[050]$} & {$[050]$} & {$[050]$} \\
\hline
\end{tabular}

Table 8. Parameters settings for GA

\begin{tabular}{|c|c|}
\hline Parameter & Value \\
\hline Chromosomes & 20 \\
Evaluations number & $10^{4}$ \\
Genes & 3 \\
Crossover rate & 0.6 \\
Crossover type & Two points \\
Mutation rate & 0.01 \\
\hline
\end{tabular}

The good results in term of fitness have led to obtain the optimal gains of the PID controller. These values have been used to control the inverted pendulum. The angle errors of the pole are represented in Figure. 8.

The gains obtained from GHS have canceled the overshoot and reduced the settling time. Those of HS have caused an overshoot of $15 \%$.

The performances obtained from GHS are the result of the good optimization process and come to confirm the statistical analysis.

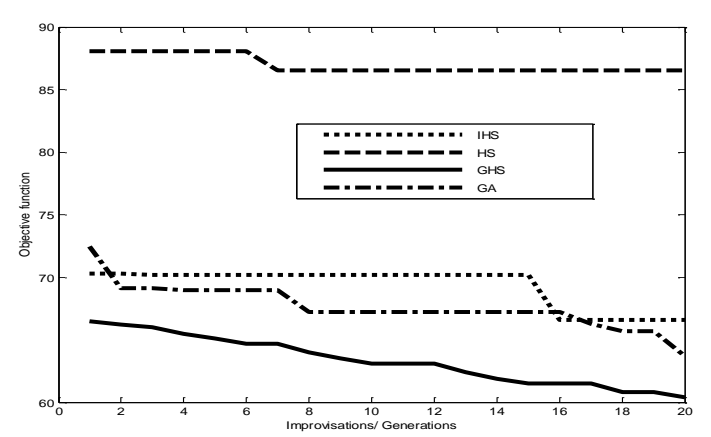

Figure 8. Objective function values for the harmony search variants and $\mathrm{GA}$ 


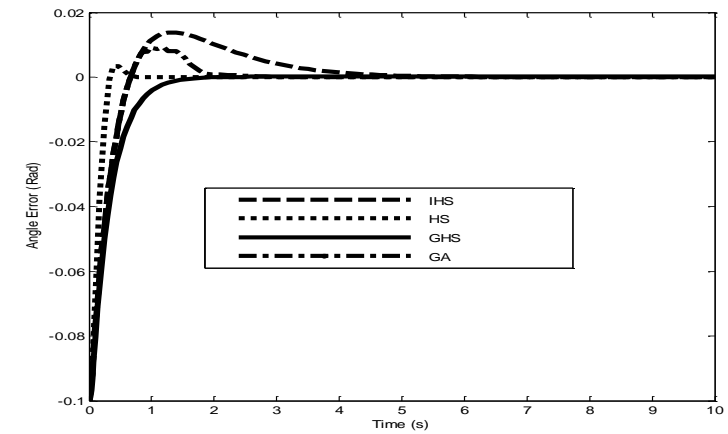

Figure 9. Angle errors for inverted pendulum (HS, GHS, IHS and GA)

For comparison purpose, we run each algorithm 10 times. For each run, the same start harmonies was used in the same conditions (improvisations number and harmony size) for GA, HS, GHS and IHS. We measured the minimum costs (Objective function for HS variants and fitness for GA). Comparison results are in Table.9.

From Table.9, GHS gives good results even with different runs and different start harmonies, this is due to the hybridization with PSO witch avoid local optima and improve the HS. These results are detailed in Table.10 where it is clear that GHS optimal gains enhance the system performances (Rising time $T_{r}$, Settling time $T_{\mathrm{s}}$ and maximum overshoot $\mathrm{O}_{\max }$ ) where GHS gets better results then GA in all the runs for rising time, in 9 runs for settling time and in 7 runs for overshoot (See Table.10).

\section{Conclusion}

We presented in this paper an overview of the harmony search algorithm and its main variants. These algorithms were evaluated using two nonparametric tests with 10 benchmark problems of function optimization: Wilcoxon test and Friedman test with its post-hoc procedures. The results of the statistical analysis highlights the GHS algorithm as the first ranked against the rest of the considered harmony search variants.

A real world application of the GHS and HS to tune PID controller gains for an inverted pendulum was also presented giving good results for GHS algorithm when compared to genetic algorithms confirming the results of the statistical analysis.

This work could be improved by considering other statistical nonparametric tests like Quade test or/ and hybridize the harmony search with other evolutionary algorithm to enhance its diversification process. The harmony search and its variants could also be applied to optimize more complex systems in dimension and ranges.

Table 9. Minimum costs for GHS,IHS, HS and GA in 10 runs.

\begin{tabular}{|c|c|c|c|c|}
\hline Run & Min HS & Min GHS & Min IHS & Min GA \\
\hline 1 & 71.5 & 63.72 & 69.41 & 65.98 \\
\hline 2 & 58.97 & 56.03 & 60.31 & 56.74 \\
\hline 3 & 56.44 & 52.88 & 58.25 & 55.08 \\
\hline 4 & 53.83 & 53.76 & 58.22 & 55.11 \\
\hline 5 & 57.15 & 55.11 & 59.57 & 60.64 \\
\hline 6 & 61.56 & 58.08 & 62.41 & 61.78 \\
\hline 7 & 53.97 & 51.79 & 57.98 & 56.35 \\
\hline 8 & 59.21 & $\mathbf{5 5 . 6 4}$ & 62.54 & 64.88 \\
\hline 9 & 55.00 & $\mathbf{4 9 . 2 3}$ & 56.20 & 52.37 \\
\hline 10 & 68.31 & $\mathbf{6 2 . 6 5}$ & 67.74 & 66.93 \\
\hline
\end{tabular}

Table 10. Performance of PID controller tuned by (GHS, GA).

\begin{tabular}{|c|c|c|c|c|c|c|c|c|}
\hline \multirow{2}{*}{ Run } & \multicolumn{5}{|c|}{$\boldsymbol{G H} \boldsymbol{~}$} & \multicolumn{4}{c|}{$\boldsymbol{G A}$} \\
\cline { 2 - 9 } & Obj func & $T_{r}(s)$ & $T_{s}(s)$ & $\boldsymbol{O}_{\max }$ & Min fitn & $T_{r}(s)$ & $T_{s}(s)$ & $O_{\max }$ \\
\hline 1 & $\mathbf{6 3 . 7 2}$ & $\mathbf{0 . 0 5 1}$ & $\mathbf{0 . 1 6 0}$ & $\mathbf{0 . 0 1 2}$ & 65.98 & 0.056 & 0.465 & 0.014 \\
\hline 2 & $\mathbf{5 6 . 0 3}$ & 0.064 & 0.175 & $\mathbf{0 . 0 1 2}$ & 56.74 & $\mathbf{0 . 0 5 8}$ & $\mathbf{0 . 1 5 8}$ & 0.014 \\
\hline 3 & $\mathbf{5 2 . 8 8}$ & $\mathbf{0 . 0 5 4}$ & $\mathbf{0 . 1 9 4}$ & $\mathbf{0 . 0 1 4}$ & 55.08 & 0.059 & 0.783 & 0.018 \\
\hline 4 & $\mathbf{5 3 . 7 6}$ & $\mathbf{0 . 0 5 3}$ & $\mathbf{0 . 1 7 2}$ & $\mathbf{0 . 0 1 1}$ & 55.11 & 0.056 & 0.175 & 0.020 \\
\hline 5 & $\mathbf{5 5 . 1 1}$ & 0.064 & $\mathbf{0 . 1 7 8}$ & $\mathbf{0 . 0 1 2}$ & 60.64 & $\mathbf{0 . 0 5 5}$ & 0.730 & 0.020 \\
\hline 6 & $\mathbf{5 8 . 0 8}$ & $\mathbf{0 . 0 6 5}$ & $\mathbf{0 . 2 0 8}$ & 0.015 & 61.78 & 0.077 & 0.301 & $\mathbf{0 . 0 0 8}$ \\
\hline 7 & $\mathbf{5 1 . 7 9}$ & $\mathbf{0 . 0 6 4}$ & $\mathbf{0 . 1 8 0}$ & $\mathbf{0 . 0 1 2}$ & 56.35 & 0.068 & 0.117 & 0.014 \\
\hline 8 & 55.64 & 0.064 & 0.191 & 0.014 & $\mathbf{5 4 . 8 8}$ & $\mathbf{0 . 0 6 1}$ & $\mathbf{0 . 1 6 7}$ & $\mathbf{0 . 0 1 3}$ \\
\hline 9 & $\mathbf{4 9 . 2 3}$ & $\mathbf{0 . 0 5 6}$ & $\mathbf{0 . 1 9 7}$ & $\mathbf{0 . 0 1 0}$ & 52.37 & 0.058 & 0.360 & 0.011 \\
\hline 10 & $\mathbf{6 2 . 6 5}$ & $\mathbf{0 . 0 6 5}$ & $\mathbf{0 . 1 5 8}$ & $\mathbf{0 . 0 1 2}$ & 66.93 & 0.068 & 0.164 & 0.013 \\
\hline
\end{tabular}




\section{References}

[1] K. Y. Lee and M. A. El-Sharkawi, Modern Heuristic Optimization Techniques: Theory and Applications to Power Systems, Wiley-IEEE Press, 2008.

[2] Z. W.Geen ,J.H.Kim and G.V.Loganathan,: “A New Heuristic Optimization Algorithm:Harmony Search“, Simulation 76, pp.60-69, 2001,

[3] X. Z. Gao, V. Govindasamy, H. Xu, X. Wang, and K. Zenger, "Harmony Search Method: Theory and Applications," Computational Intelligence and Neuroscience, vol. 2015,pp 1$10,2015$.

[4] S.L. Kang, Z.W. Geem, "A new structural optimization method based on the harmony search algorithm", Comput. Struct. vol 82 (910), pp.781-798, 2004.

[5] P. Karthigeyan, M. Senthil Raja, R. Hariharan, S. Prakash, S. Delibabu, R. Gnanaselvam, Comparison of Harmony Search Algorithm, Improved Harmony Search Algorithm with Biogeography Based Optimization Algorithm for Solving Constrained Economic Load Dispatch Problems, Procedia Technology, Volume 21, pp 611-618, 2015.

[6] K.S. Lee, Z.W. Geem," A new meta-heuristic algorithm for continues engineering optimization: harmony search theory and practice", Comput. Meth. Appl. Mech. Eng. vol 194, pp. 3902-3933, 2004.

[7] M. Mahdavi, Fesanghary, M., Damangir, E.: An improved harmony search algorithm for solving optimization problems. Applied Mathematics and Computation vol 188, pp 1567-1579, 2007.

[8] M.G.H. Omran, Mahdavi, M." Global-best harmony search". Applied Mathematics and Computation vol 198, pp.643-656, 2008.

[9] C.M. Wang, Huang, Y.F. " Self-adaptive harmony search algorithm for optimization". Expert Systems with Applications, Vol 37(4), pp 2826-2837, 2010.

[10] O. Hasancebi, F.Erdal, M.P.Saka , " An adaptive harmony search method for structural optimization", Journal of Structural Engineering, vol 137(4), pp 419-431, 2010.

[11] J. J., Liang, B-Y. Qu, P. N. Suganthan, and A.G. Hernndez-Daz, " Problem Definitions and Evaluation Criteria for the CEC 2013 Special Session and Competition on Real-Parameter Optimization", Technical Report 201212, Computational Intelligence Laboratory,
Zhengzhou University, Zhengzhou China and Technical Report, Nanyang Technological University, Singapore, 2013.

[12] S., Garcia, A. Fernndez, J. Luengo and F. Herrera,. "Advanced nonparametric tests for multiple comparisons in the design of experiments in computational intelligence and data mining: Experimental analysis of power", Information Sciences, vol 180, pp. 2044-2064, 2010.

[13] J. H., Zar, Biostatistical Analysis, Prentice Hall. , 2009.

[14] D.H. Wolpert, W.G. Macready, No free lunch theorems for optimization, IEEE Transactions on Evolutionary Computation, vol 1(1), pp 67$82,1997$.

[15] P. Wang and D. P. Kwok, "Optimal design of PID process controllers based on genetic algorithms," Control Engineering Practice, vol. 2(4), pp. 641-648, 1994.

[16] M. Friedman, "A comparison of alternative tests of signiffcance for the problem of $\mathrm{m}$ rankings". Annals of Mathematical Statistics, vol 11, pp. 86-92, 1940.

[17] M. Salem, M.F. Khelfi, "Application of Biogeography based optimization in tuning a PID controller for nonlinear systems “, International Conference on Complex Systems (ICCS), November 4-6, 2012, Agadir, Morocco

[18] D. Shilane, J. Martikainen, S. Dudoit, S.J. Ovaska, A general framework for statistical performance comparison of evolutionary computation algorithms, Information Sciences, vol 178 (14) , pp 2870-2879, 2008. 